\title{
ON CONGRUENCE CONDITIONS FOR PRIMALITY
}

\author{
Sherry Gong \\ Departments of Mathematics and Physics, Harvard University \\ sgong@fas.harvard.edu \\ Scott Duke Kominers ${ }^{1}$ \\ Department of Economics, Harvard University, and Harvard Business School \\ kominers@fas.harvard.edu, skominers@gmail.com
}

Received: 5/22/09, Revised: 2/28/10, Accepted: 3/7/10, Published: 7/16/10

\begin{abstract}
For any $k \geq 0$, all primes $n$ satisfy the congruence $n \sigma_{k}(n) \equiv 2 \bmod \varphi(n)$. We show that this congruence in fact characterizes the primes, in the sense that it is satisfied by only finitely many composite $n$. This characterization generalizes the results of Lescot and Subbarao for the cases $k=0$ and $k=1$. For $0 \leq k \leq 14$, we enumerate the composite $n$ satisfying the congruence. We also prove that any composite $n$ which satisfies the congruence for some $k$ satisfies it for infinitely many $k$.
\end{abstract}

\section{Introduction}

Lescot [1] and Subbarao [2] showed that, for $k \in\{0,1\}$, the congruence

$$
n \sigma_{k}(n) \equiv 2 \bmod \varphi(n),
$$

in some sense characterizes the set $\mathcal{P}$ of primes. Specifically, they respectively showed the $k=0$ and $k=1$ cases of the following theorem.

Theorem 1. For $k \in\{0,1\}$ and $n \in \mathbb{N}$, the congruence (1) holds if and only if $n \in \mathcal{P} \cup\{1\} \cup S_{k}$, where $S_{0}=\{4,6,14\}$ and $S_{1}=\{4,6,22\}$.

Here, $\sigma_{k}$ and $\varphi$ respectively denote the divisor and totient functions, defined by

$$
\begin{gathered}
\sigma_{k}(n)=\sum_{d \mid n} d^{k}=\prod_{i=1}^{r} \frac{p_{i}^{\left(\alpha_{i}+1\right) k}-1}{p_{i}^{k}-1} \\
\varphi(n)=\mid\{d \in \mathbb{N}: 1 \leq d<n \text { and }(d, n)=1\} \mid=\prod_{i=1}^{r} p_{i}^{\alpha_{i}-1}\left(p_{i}-1\right),
\end{gathered}
$$

for $k \geq 0$ and $\mathbb{N} \ni n=p_{1}^{\alpha_{1}} \cdots p_{r}^{\alpha_{r}}$ (with the $p_{i} \in \mathcal{P}$ distinct).

\footnotetext{
${ }^{1}$ Corresponding author.
} 
It is clear that if $n \in \mathcal{P}$, then $\sigma_{k}(n)=n^{k}+1$ and $\varphi(n)=n-1$. In this case, we have

$$
n \sigma_{k}(n) \equiv n\left(n^{k}+1\right) \equiv 2 \bmod (n-1),
$$

as $p \equiv 1 \bmod (p-1)$. We therefore see that, for any $k \geq 0$, all $n \in \mathcal{P}$ satisfy (1).

In this note, we present the following result generalizing the reverse direction of Theorem 1.

Theorem 2. For any $k \geq 0$, let $S_{k}$ be the set of composite $n \in \mathbb{N}$ satisfying (1). Then,

(i) $S_{k} \subset 2 \mathcal{P}$,

(ii) $S_{k}$ is finite, and

(iii) the maximal element of $S_{k}$ is at most $2^{k+3}+6$.

We prove Theorem 2 in Section 2. Then, in Section 3, we enumerate the sets $S_{k}$ for $0 \leq k \leq 14$. There, we also prove that any $n \in \mathbb{N}$ which appears in $S_{k}$ for some $k \geq 0$ appears in infinitely many of the sets $\left\{S_{k^{\prime}}\right\}_{k^{\prime}=0}^{\infty}$.

\section{Proof of Theorem 2}

We suppose that $n \in S_{k}$, i.e., that $n$ is composite and satisfies (1). Upon writing $n=2^{\alpha} p_{1}^{\alpha_{1}} \cdots p_{r}^{\alpha_{r}}$ with the $p_{i} \in \mathcal{P}$ distinct and odd, we have

$$
\varphi(n)=2^{\alpha-1} \prod_{i=1}^{r} p_{i}^{\alpha_{i}-1}\left(p_{i}-1\right)
$$

For any $i(1 \leq i \leq r)$ such that $\alpha_{i}>1$, we see from the expression (2) that $p_{i} \mid \varphi(n)$. Since clearly $p_{i} \mid n$, the congruence (1) then gives that $p_{i} \mid 2$-an impossibility. Thus, we must have $\alpha_{i}=1$. An analogous argument shows that $\alpha \in\{1,2\}$.

Now, as $p_{i}-1$ is even for each $i(1 \leq i \leq r)$, we see from (2) that $2^{r} \mid \varphi(n)$. Furthermore, the expression

$$
\begin{aligned}
\sigma_{k}(n) & =\frac{2^{(\alpha+1) k}-1}{2^{k}-1} \prod_{i=1}^{r} \frac{p_{i}^{\left(\alpha_{i}+1\right) k}-1}{p_{i}^{k}-1} \\
& =\frac{2^{(\alpha+1) k}-1}{2^{k}-1} \prod_{i=1}^{r} \frac{p_{i}^{2 k}-1}{p_{i}^{k}-1}=\frac{2^{(\alpha+1) k}-1}{2^{k}-1} \prod_{i=1}^{r}\left(p_{i}^{k}+1\right)
\end{aligned}
$$


yields that $2^{r} \mid \sigma_{k}(n)$ since $2 \mid p_{i}^{k}+1$. We then obtain from the congruence (1) that $2^{r} \mid 2$, whence we see that $r \leq 1$.

Since $n$ is composite, we are left with only two possibilities: $n=2 p$ (with $p \in \mathcal{P}$ ) or $n=4 p$ (with $p \in \mathcal{P}$ ). The second case is impossible, as when $n=4 p$ we have (1) and $4 \mid \varphi(n)$, together implying $4 \mid 2$; the fact that $S_{k} \subset 2 \mathcal{P}$ follows. In the first case, if $p=2$, then $n=4 \leq 2^{k+3}+6$. If $p$ is odd, then we have $n \sigma_{k}(n)=2 p\left(2^{k}+1\right)\left(p^{k}+1\right)$ and $\varphi(n)=p-1$. Because $p \equiv 1 \bmod (p-1)$, we see that

$$
n \sigma_{k}(n) \equiv 2 p\left(2^{k}+1\right)\left(p^{k}+1\right) \equiv 4\left(2^{k}+1\right) \bmod (p-1) .
$$

Combining (1) and (4), we see that

$$
(p-1) \mid 2^{k+2}+2
$$

As $n=2 p$ and (5) implies that $p \leq 2^{k+2}+3$, we have the stated bound on the size of $n \in S_{k}$; the finitude of $S_{k}$ follows.

\section{The Sets $S_{k}$}

Table 1 presents the exceptional sets $S_{k}$ for $0 \leq k \leq 14$. It is clear that $4,6 \in S_{k}$ for each $k \geq 0$, since $\varphi(4)=\varphi(6)=2$, and $4 \sigma_{k}(4)$ and $6 \sigma_{k}(6)$ are even for all $k$. Beyond this observation, however, the behavior of the sets $S_{k}$ appears to be quite erratic.

Nonetheless, we obtain the following partial characterization result for the $S_{k}$.

Corollary 3. If $n \in \mathbb{N}$ is in $S_{k}$ for some $k \geq 0$, then it is in infinitely many of the sets $\left\{S_{k^{\prime}}\right\}_{k^{\prime}=0}^{\infty}$.

Proof. It is easily seen in the proof of Theorem 2 that $n \in S_{k}$ if and only if $n=2 p$ for $p \in \mathcal{P}$ satisfying

$$
(p-1) \mid\left(2^{k+2}+2\right),
$$

or equivalently,

$$
\frac{p-1}{2} \mid\left(2^{k+1}+1\right)
$$

But this means that $2^{k+1} \equiv-1 \bmod \frac{p-1}{2}$, hence $2^{2 k+2} \equiv 1 \bmod \frac{p-1}{2}$. It follows that we have

$$
2^{(2 j+1)(k+1)-1}=2^{(2 k+2) j+k} \equiv 2^{k} \bmod \frac{p-1}{2}
$$




\begin{tabular}{l|l}
$k$ & $S_{k}$ \\
\hline 0 & $\{4,6,14\}$ \\
1 & $\{4,6,22\}$ \\
2 & $\{4,6,14,38\}$ \\
3 & $\{4,6\}$ \\
4 & $\{4,6,14,46,134\}$ \\
5 & $\{4,6,22,262\}$ \\
6 & $\{4,6,14\}$ \\
7 & $\{4,6\}$ \\
8 & $\{4,6,14,38\}$ \\
9 & $\{4,6,22,166\}$ \\
10 & $\{4,6,14,2734\}$ \\
11 & $\{4,6\}$ \\
12 & $\{4,6,14\}$ \\
13 & $\{4,6,22,118,454\}$ \\
14 & $\{4,6,14,38,46,134,398,3974,14566\}$
\end{tabular}

Table 1: The exceptional sets $S_{k}(0 \leq k \leq 14)$

for any $j \in \mathbb{N}$. Then, for any $k^{\prime} \in\{(2 j+1)(k+1)-1\}_{j=1}^{\infty}$, we have

$$
(p-1) \mid\left(2^{k^{\prime}+2}+2\right)
$$

hence $n \in S_{k^{\prime}}$. We have therefore produced infinitely many $k^{\prime}$ such that $n \in S_{k^{\prime}}$.

\section{References}

[1] P. Lescot, A characterisation of prime numbers, The Mathematical Gazette 80 (1996), no. 488, 400-401. 
[2] M. V. Subbarao, On two congruences for primality, Pacific Journal of Mathematics 52 (1974), 261-268. 